\title{
Time domain analysis of partial discharges envelope in medium voltage XLPE cables
}

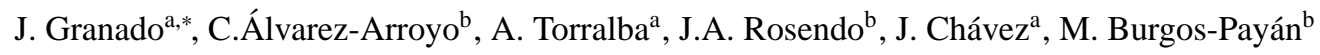 \\ Universidad de Sevilla. Descubrimientos s/n. 41092-Sevilla (Spain) \\ ${ }^{a}$ Department of Electronic Engineering \\ ${ }^{b}$ Department of Electrical Engineering
}

\begin{abstract}
Partial discharge (PD) analysis has been widely used to detect premature degradation of power cables. Although there are recognized techniques for $\mathrm{PD}$ analysis, there is still lack of knowledge about measuring and modeling this phenomenon.

This paper proposes a new model based on time domain parameters of the PD signal envelope. The proposed variables (time duration, and rising and falling slopes of the envelopes), together with conventional PD analyses focused on PD amplitude and phase resolved patterns, will provide a better understanding of this phenomenon. Based on this model, a new technique to reshape the PD signal envelope is also proposed that compensates the negative effect of dispersion in the location techniques that estimate the time of arrival (TOA).

Experimental results were obtained in the lab, where a power cable has been artificially damaged in order to produce PD. To this end, a specific PD on-line acquisition system has been developed. In the first set of experiments, we illustrate how the cable attenuates and disperses the PD signal envelope. In the second one, we show how the proposed variables are useful to distinguish among different types of PD sources. Finally, an accurate estimation of the PD source location is achieved by measuring the time of arrival of the PD signal envelopes at both cable ends, and reshaping one of the signal captured. This technique improves the accuracy of the estimated location of the PD sources, both in simulation and in experimental results.
\end{abstract}

Keywords: Partial discharges, power cables, cross-linked polyethylene insulation, time domain analysis, time domain measurements, fault location.

\section{Introduction}

Medium voltage (MV) underground cables are widely used in power networks. Many of these cables, in service for extended periods of time, use cross-linked polyethylene (XLPE) as insulation material. During manufacturing, installation or normal operation, cable insulation defects result in the occurrence of partial discharges (PDs). Additionally, PDs will gradually degrade and erode the dielectric materials, eventually leading to insulation breakdown. Therefore, PD activity is one of the indicators used to assess the state of the insulation system [1]. It is well know that XLPE is less resistant to partial discharges than other materials [2].

A reliable monitoring system requires a comprehensive knowledge of PD signal characteristics, including PD acquisition, detection, location and propagation characteristics, etc. Propagation characteristics in MV XLPE cables are studied in $[3,4,5,6]$. Different test circuits and techniques are compiled to IEC 60270 [7] and IEEE 400 [8] standards. However, trends in MV maintenance involve on-line, on-site, and longterm monitoring systems that are gradually replacing traditional

\footnotetext{
*Corresponding author: Tel./Fax +34954481303/+34954487373

Email addresses: j_granado@us.es (J. Granado),

cesaralvarez@us.es (C.Álvarez-Arroyo), torralba@us.es (A. Torralba), rosendo@us.es (J.A. Rosendo), chavez@zipi.us.es (J. Chávez), mburgos@us.es (M. Burgos-Payán)
}

off-line techniques (e.g. [9], [10]) to asses the insulation state of power cables.

In this paper we propose a new acquisition system using an alternative method. We use broadband power line communications current transformer (PLC-CT), already preinstalled in some MV cables. The captured information is off-line processed to detect the envelope of the PD signal, from which a set of related variables are extracted and used to tune a new time domain model. This paper also includes three case studies showing that the time domain analysis of the PD envelopes is a useful tool to better understand the PD phenomenon. The first one shows that the PD signal envelope is not only attenuated, but also dispersed, along the cable propagation. The second case study illustrates that the PD source can be discriminated using the proposed time domain model. To this end, four experiments were carried out in a MV cable, showing that different types of PD yield to different distributions of the proposed time domain variables. In the third case study, we propose a method to compensate the pulse dispersion due to cable propagation. This dispersion, that is responsible for degrading the performance of PD location techniques $[6,11]$, has been measured and a simple method is proposed to compensate for its negative effects, thus improving the overall results.

This paper is organized in following 4 additional sections. Section 2 describes the test setup including both the MV infrastructure and the acquisition system. Section 3 describes a 


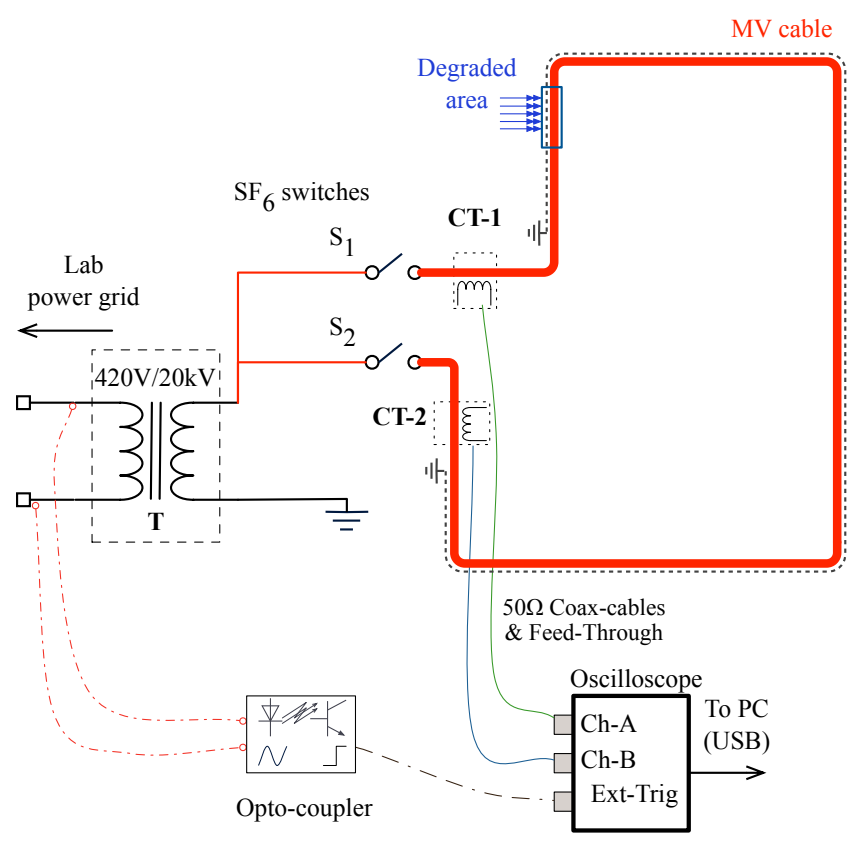

(a)

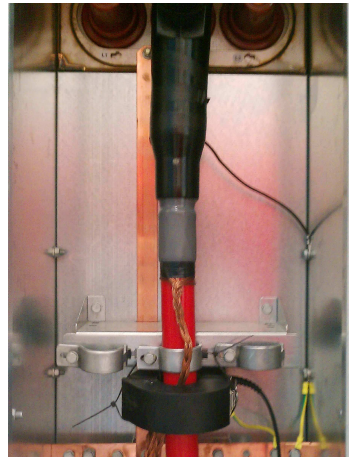

(b)

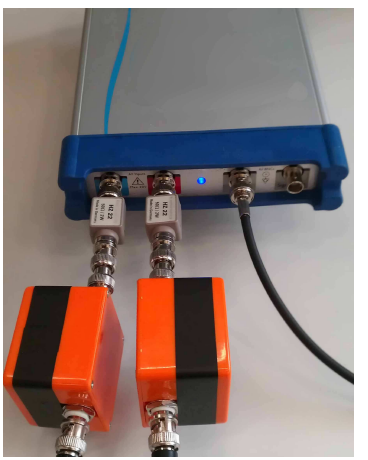

(c)
Figure 1: Test bench description: (a) schematic of MV components and acquisition system (partial discharges are artificially provoked in a degraded area close to switch $\mathrm{S}_{1}$ ); (b) CT installed in one of the cable ends; and (c) protection units, feed-through impedances, and the programmable oscilloscope.

new PD model based on the time domain attributes of the partial discharge envelope. Section 4 illustrates the proposed time domain analysis by means of three case studies. Finally, some conclusions are drawn in section 5 .

\section{Test bench description}

\subsection{Medium voltage installation setup}

The MV test setup is briefly described in Figure 1(a). A 340 $\mathrm{m}$ long XLPE cable was stressed at operating conditions (18 $\mathrm{kV}$ ) according to standard IEEE 400 [8]. A dry transformer (T) elevated the low incoming mains voltage to medium voltage. In the MV side two $\mathrm{SF}_{6}$ circuit breakers (labeled as $\mathrm{S}_{1}$ and $\mathrm{S}_{2}$, respectively) allowed the cable to be fed through either end or both ends at the same time.

\subsection{Signal coupling}

Two inductive broadband couplers (current transformers CTs) labeled as CT-1 and CT-2 in Figure 1(a), respectively, were clamped at both MV cable ends once the cable ground was retracted (see Figure 1(b)). These components are responsible for detecting PDs. The selected CT was an inexpensive inductive signal coupler used for broadband power line commutation (PLC) over the MV grid.

In order to determine how much the current transformers attenuate the frequency components of the captured PD, the insertion losses of the CTs are measured using a vector network analyzer. Figure 2 shows the attenuation by the selected CT in the range [1,100] MHz. According to standard IEC 60270 [7], the selected CT was an ultra-wide band (UWB) coupler. Figure 2 shows that the lowest frequency components were attenuated up to $12.92 \mathrm{~dB}$, whereas the highest frequency components were attenuated by a maximum of $12.45 \mathrm{~dB}$. In fact, according to the experimental results measured with a vector network analyzer, the XLPE cable exhibited an attenuation of $0.17 \mathrm{~dB} / \mathrm{m}$ at $50 \mathrm{MHz}$. This attenuation increased up to $0.29 \mathrm{~dB} / \mathrm{m}$ at 100 MHz. Therefore, in a $300 \mathrm{~m}$ long cable, components greater that $50 \mathrm{MHz}$ are almost extinct. Of note, partial discharges with frequency components lower than $1 \mathrm{MHz}$ cannot be measured using the selected CTs.

Other commercial products have also been studied. On the one hand, narrow-band sensors such as Rogowski coils [10] are unable to measure important spectral components located at high frequencies. On the other hand, in [9], an ultra wide band sensor is proposed that covers from $300 \mathrm{MHz}$ to $800 \mathrm{MHz}$. Due to the strong attenuation at these frequency components, the PD capturing area is restricted to the area around the cable termination where the UWB sensor is attached. The selected CT is a tradeoff between ultra wide-band and narrow-band sensors.

\subsection{Acquisition system}

The current transformers outputs were connected to an oscilloscope with a 2 ns sampling time $\left(T_{S}\right)$ and $250 \mathrm{MHz}$ bandwidth. A programmable oscilloscope controlled from a PC was used. CTs were connected to the oscilloscope by means of $50 \Omega$ coaxial cables. Feed-through impedances matched the impedances of the coupler-cable-oscilloscope link in order to avoid signal reflections. A protection unit was designed ad-hoc to protect the measuring equipment (see Figure (1c)).

Data acquisition was synchronized to the MV zero crossing by means of an optical coupler directly connected to transformer T. The optocoupler provided a low jitter square signal whose falling edge was synchronized to the positive slope zero crossing of the power voltage. It was used as a trigger signal for the oscilloscope.

A Linux based PC was used to control, store and off-line post-process the captured data. A set of programs was especially designed to properly perform these tasks. Data acquisition was carried out according to the following operation mode:

- One oscilloscope channel captured the signal from CT-1, while the other channel captured the signal from CT-2. 


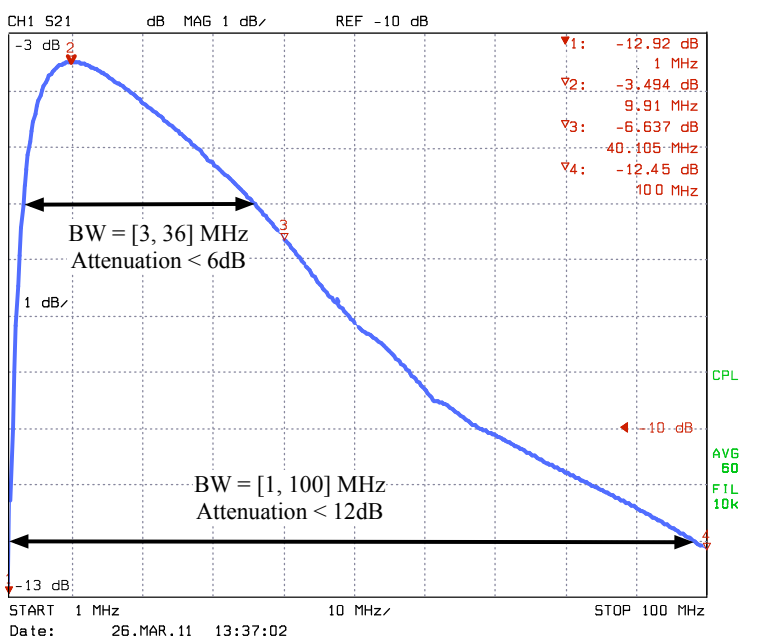

Figure 2: Insertion losses of the selected CT versus frequency in the range [1, 100] MHz. The horizontal axis is divided into constant intervals of $10 \mathrm{MHz}$ per division whereas the vertical axis is shown with $1 \mathrm{~dB}$ per division in the interval $[-13,-3] \mathrm{dB}$.

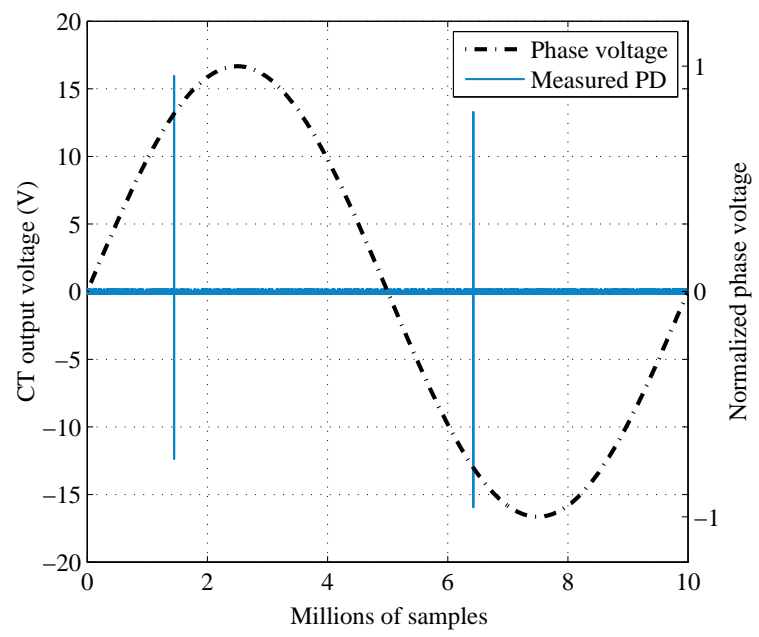

Figure 3: Example of PD capture. Using a sample time of $2 \mathrm{~ns}, 10$ millions of samples are captured $(20 \mathrm{~ms})$. Phase voltage and the CT output signals are shown.

- The duration of all the measurements was fixed to $20 \mathrm{~ms}$ (one cycle of the power voltage), externally triggered using the optical coupler.

- All measurements were done using the maximum available sampling frequency in dual mode (sampling time $T_{s}$ $=2 \mathrm{~ns}$ ) leading to 10 million samples per channel.

- Data acquisition was automatically executed from the PC using software tools such as the crontab utility. Once the experiment was finished, the data were off-line processed.

- Both, at the beginning and at the end of each experiment, the power signal was captured, analyzed and used to detect the existence of impulsive noise or any other kind of disturbing signals in the laboratory power grid.

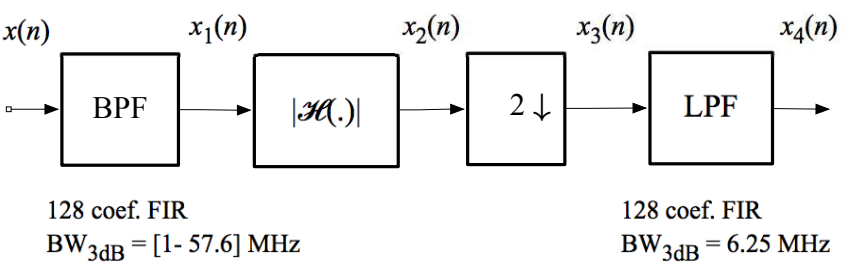

Figure 4: Description of the proposed envelope detector: $x(n)$ is the captured signal, $|\mathscr{H}|$ is the absolute value of the Hilbert transform, and the resulting envelope estimation is $x_{4}(n)$.

Figure 3 shows an example of PD captured with the proposed acquisition system. Notice that, as the captures are synchronized with the power voltage, the PD phase is known.

A set of MATLAB scripts for off-line processing of the raw data captured by the oscilloscope were developed. Firstly, the original file format is converted to an internal database format. Secondly, if a PD is detected, the signal envelope is estimated according to the technique proposed in the following section. Finally, a set of variables are obtained and used to tune the PD's envelope time domain model.

\section{Time domain analysis}

\subsection{PD envelopes estimation}

Figure 4 describes the scheme proposed for off-line detection of the PD's envelope. Firstly, these samples are bandpass filtered (block BPF) to reject both low and high frequency components. After studying the frequency components of the PD signal, signal components below $1 \mathrm{MHz}$ were found to be mainly due to conducted electromagnetic interferences whose sources can be found in the laboratory environment. The high frequency components were mainly due to FM broadcast. Note, the MV cable is large enough, $340 \mathrm{~m}$ approximately, to behave as an antenna for high frequency carriers. The BPF has been implemented using a finite impulse response (FIR) filter with 128 coefficients and zero-phase response.

The envelope computation was performed by means of the Hilbert transform. In Figure 4 signal $x_{2}(n)$ is the magnitude of the Hilbert decomposition, that is, the envelope of $x_{1}(n)$. Finally, a low pass frequency filter (LPF) selects the envelope, located in the low part of the spectrum, rejecting the high frequency components. The LPF was implemented with a 128 coefficient FIR filter with zero-phase response, and a $3 \mathrm{~dB}$ cutoff frequency of $6.25 \mathrm{MHz}$. To facilitate the implementation, its input signal, $x_{2}(n)$, is decimated by a factor of two.

Figure 5 shows an example of the envelope detection. Let $s_{\mathrm{CT}-1}(t)$ and $s_{\mathrm{CT}-2}(t)$ be the signals captured by CT- 1 and CT2 , respectively. We call the $s_{\mathrm{CT}-1}(t)$ the near-end signal as it is captured close to the degradation area where the PDs are likely to appear (see Figure 1). In the same way, the signal captured by CT-2, at the cable far end, is called the far-end signal $\left(s_{\mathrm{CT}-2}(t)\right)$. Both signals are sampled using the digital oscilloscope and synchronized using the same trigger signal (coming from the optocoupler connected to the lab mains). Figure 5 shows the estimated envelopes, labeled as $x_{4}$, of the near and far ends signals, 


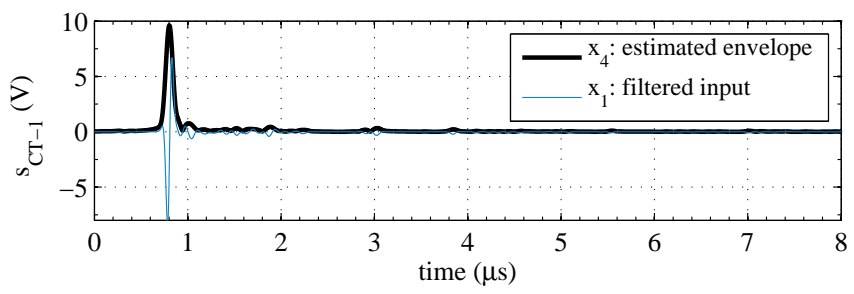

(a)

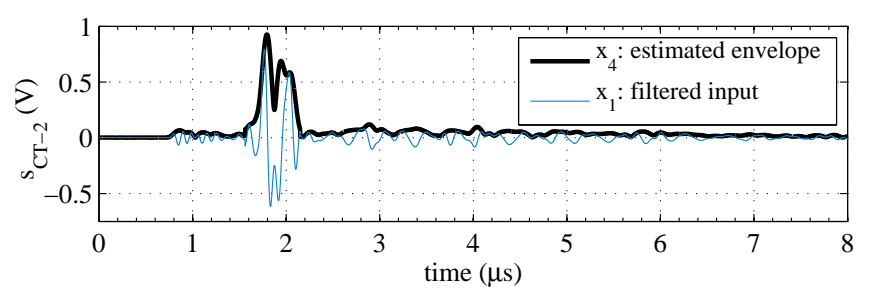

(b)

Figure 5: Example of the PD envelope of captured signals: (a) near end (close to the degradation area $\left.s_{\mathrm{CT}-1}(t)\right)$, and (b) far end $\left(s_{\mathrm{CT}-2}(t)\right)$. In both subplots the intermediate signal $\left(x_{1}\right)$, and the output signal envelope $\left(x_{4}\right)$ versus time are shown.

respectively. Figure 5 also depicts an intermediate signal, $x_{1}$ (see Figure 4), confirming that a correct envelope detection is being performed.

The envelopes depicted in Figure 5 show that the far-end signal envelope is attenuated, delayed, and dispersed when compared to the near-end signal envelope. The maximum peak value of the envelope of signal $s_{\mathrm{CT}-1}(t)$ is high $(10 \mathrm{~V})$ whereas the envelope of the far-end signal $s_{\mathrm{CT}-2}(t)$ exhibit a max value of $1 \mathrm{~V}$, and it is approximately delayed $2 \mu \mathrm{s}$. In this example, and assuming that the PD is generated in the degradation area close to the CT-1, it can be deduced that the propagation velocity of the envelope is roughly $170 \mathrm{~m} / \mu \mathrm{s}$. It can be shown that the far-end signal envelope is also dispersed.

\subsection{Proposed time domain model}

Before computing the envelope, the PD signal has to be discriminated from background noise. To this end, we selected the following strategy: a detection threshold $\left(D_{T H}\right)$ was defined and the captured samples were considered a PD capture only when more than $M$ samples are above this threshold. Otherwise, they are considered to be background noise. Proper values for $M$ and $D_{T H}$ have been chosen for each experiment. The samples classified as being part of a PD capture are processed in order to estimate the following time domain variables.

\subsubsection{Maximum amplitude (MA), time deviation $(\sigma)$, and phase (Pha)}

The envelope of the PD signal measured at the cable end (far enough from the source) can be modeled as a Gaussian pulse with amplitude $M A$, and width or deviation $\sigma$ :

$$
x(t)=M A \exp \left(-t^{2} / 2 \sigma^{2}\right)
$$

The acquisition system was synchronized with the power voltage by means of an optical coupling. This reference is used to compute the phase angle $(P h a)$ of the PD signal envelope.

Both variables, $M A$ and $\sigma$, can be used to estimate the charge associated to the PD envelope according to reference [12], also called PD magnitude [5]. Existing visualization tools provide diagrams of apparent charge versus Pha, also called phase resolved PD (PRPD) patterns, but they presents some drawbacks: a) the cable impedance depends on the frequency, specially in the proximity of the PD source where its bandwidth is wide (about a GHz); b) the maximum amplitude of the PD depends directly on the position where the signal is captured (highest frequencies are rapidly attenuated due to cable losses and, in a few hundred meters, no more than a few MHz survive the cable propagation); and c) the original pulse width is spread due to the dispersive behavior of the cable (some frequency components of the PD propagate at higher velocity than others, resulting in a relative phase shift between components that depends on the propagated distance [6]). Both dispersion and high frequencies attenuation distort the original pulse shape and, consequently, mislead the estimated apparent charge. In fact, it is accepted that time to failure cannot be predicted on the basis of discharge magnitude [13].

\subsection{2. $P D$ rising and falling slopes $(R S, F S)$}

Modeling the PD envelope using a Gaussian pulse is useful to estimate some interesting magnitudes as described in the above section, but it does not consider the skewed nature of the PD pulse [6], which also is perceived in its envelope (especially close to the source). A simple model based on the envelope slopes is proposed here. The rising slope $(R S)$ and the falling slope $(F S)$ are defined by means of a simple linear approximation around the point where the envelope reaches its standard deviation value: $M A \exp (-0.5)$. Examples of these variables in different conditions can be seen in the case studies included in the following section.

\section{Case studies}

In order to perform a more complete diagnostic of the MV cable, all of the variables described above provide additional knowledge. In this section we address three case studies where the previously defined variables are used to gain a detailed understanding of the partial discharge phenomenon. In the first case, we show how the PD signal envelope is attenuated and dispersed. Secondly, these variables are used to distinguish among different types of PD. Finally, we propose a reshaping algorithm, which improves the accuracy of the PD source location using cross-correlation of PD envelopes.

\subsection{Attenuation and dispersion}

Figure 6 shows a diagram where the maximum amplitude (MA) was drawn versus the PD pulse width $(\sigma)$, in both cable ends. This experiment was carried out using a MV cable where the dielectric was intentionally degraded. Note that the PDs detected close to the degradation area are greater, in terms of 


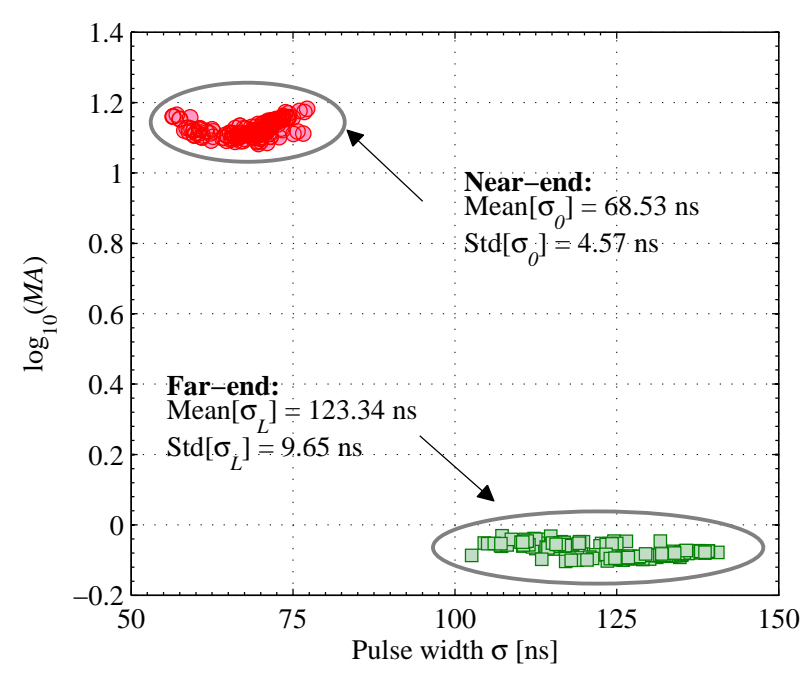

Figure 6: Envelope maximum amplitude $M A$ versus pulse width $\sigma$ diagram for a degraded cable. $\sigma_{0}$ and $\sigma_{L}$ are the widths measured at the near and far ends, respectively.

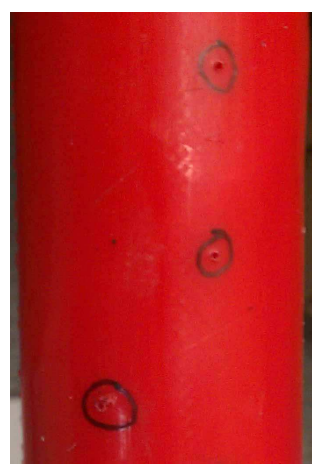

(a)

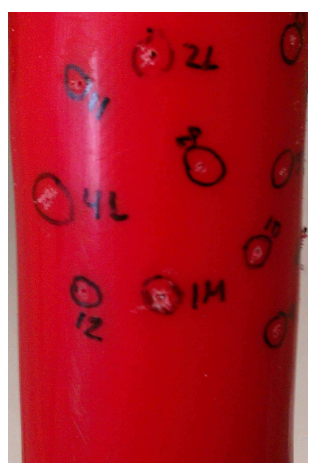

(b)
Figure 7: Artificial defects performed in the cable: (a) low degradation (used in experiment A) and (b) high degradation (experiment B).

$M A$, and narrower, in terms of $\sigma$, than those detected in the far-end. Figure 6 shows that the original signal envelope is attenuated approximately $24 \mathrm{~dB}$ and dispersed from $68 \mathrm{~ns}$ up to $123 \mathrm{~ns}$. Note that the original pulse width is not known since the signal is captured only in cable ends. However, in the test setup of the Figure 1, the cable was damaged very close to the near cable end where the signal is being captured.

\subsection{Distinguishing types of PD}

In this case study we will analyze the proposed time domain variables to discriminate among different types of PD. Four experiments have been performed:

- Experiment A: a new cable with a slightly degraded area.

- Experiment B: a new cable with a severely degraded area.

- Experiment C: a just repaired cable.

- Experiment D: a new cable with no visible defects.
Table 1: Mean value and standard deviation (mean / deviation) of the measured envelope width $(\sigma)$, rising slope $(R S)$, and falling slope $(F S)$.

\begin{tabular}{lccc} 
Exp.: & $\sigma[\mu \mathrm{s}]$ & $R S[\mathrm{~V} / \mu \mathrm{s}]$ & $F S[\mathrm{~V} / \mu \mathrm{s}]$ \\
\hline A & $67.6 / 5.4$ & $110.1 / 6.1$ & $79.6 / 10.7$ \\
B & $119.9 / 18.8$ & $157.6 / 5.7$ & $97.7 / 11.6$ \\
C & $146.4 / 10.1$ & $13.4 / 2.9$ & $6.0 / 0.8$ \\
D & $97.9 / 2.2$ & $88.9 / 4.5$ & $57.5 / 3.2$ \\
\hline
\end{tabular}

Table 2: Mean value and standard deviation (mean / deviation) of the measured phase angle $(P h a)$. It is divided between first and second power phase semiperiods.

\begin{tabular}{lll} 
Exp.: & $(P h a / \pi) 1^{\text {st }}$ & $(P h a / \pi) 2^{\text {nd }}$ \\
\hline A & $0.27 / 0.02$ & $1.30 / 0.02$ \\
B & $0.23 / 0.03$ & $1.34 / 0.03$ \\
C & $0.25 / 0.01$ & $1.31 / 0.01$ \\
D & $0.30 / 0.03$ & $1.28 / 0.03$ \\
\hline
\end{tabular}

In the experiments A and B, a new cable was degraded by introducing artificial defects in the cable insulation systems. Two cable degradation conditions have been studied: in experiment A the cable has three superficial holes in the degradation area; and the same cable has sixteen deeper holes in experiment B. Figure 7(a) and 7(b) show the photographs of the cable in experiment $\mathrm{A}$ and $\mathrm{B}$, respectively. In both cases the thermoplastic sheath, the semiconducting layer and the insulation were punctured. Hole penetration in the insulation was $2 \mathrm{~mm}$ and $5 \mathrm{~mm}$ for experiment A and B, respectively. In both tests, the MV cable was clearly degraded, however, the exact condition of the dielectric could not be determined. Since in the experiment B the holes in the cable were greater in depth and number, we assume this situation is more severe. In fact, the cable of Figure 7(b) broke down one week after the damage was done to it.

The cable was repaired following failure by cutting the damaged section and replacing the termination. Just after repairing the cable we performed the experiment $C$, and significant PD activity was observed that rapidly disappeared. This activity, did not appear in the following experiments. Finally, the experiment $\mathrm{D}$ is performed on a new cable with no visible defects.

We detected high frequency activity in the experiments. In A and $\mathrm{B}$ we are sure that the activity origin was in the degraded area, and the PD was caused by the defect introduced in cable. In experiment $\mathrm{C}$ the activity was related to the reparation process, and we think that the PD was generated in the cable termination. In these three experiments the PD is generated at one cable end (experiment $\mathrm{C}$ ), or close to a cable end (experiments $\mathrm{A}$ and $\mathrm{B}$ ), and propagated along the cable. We have measured the difference between the times of arrival at each CT from its origin, confirming that the $\mathrm{PD}$ traveled along the cable in experiment $\mathrm{A}, \mathrm{B}$, and $\mathrm{C}$. Conversely, we think that the signals captured in the experiment $\mathrm{D}$ were not generated in the cable, because the difference between the times of arrival was almost zero. The origin of the signals captured in experiment $\mathrm{D}$ must be found outside of the MV circuit, probably they were generated in the LV/MV transformer. 


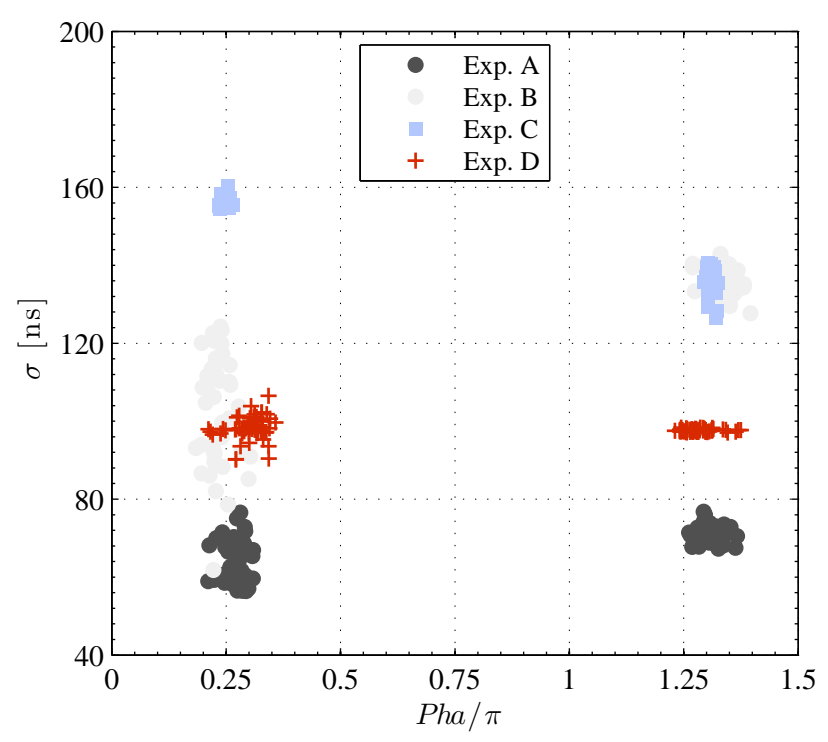

Figure 8: Envelope deviation $(\sigma) v s$. power voltage phase angle Pha.

Experimental results are graphically shown in Figure 8, 9, and 10. Tables 1 and 2 present the mean and standard deviation of the measured variables. All these variables were measured at the near-end location.

Variable $\sigma$ versus Pha is drawn in the diagram of Figure 8, where Pha was normalized by $\pi$. The most important result is related to the envelope width $\sigma$. Comparing experiment $\mathrm{A}$ and $\mathrm{B}$, we observe that the envelope widths are different, although the origin of the PD is similar. We think that, in experiment B, several PDs are being produced almost simultaneously in the degraded area, but with a few nanoseconds delay between them. The superposition of these PDs is responsible for the envelope spreading.

Regarding the variable Pha, we can see in Figure 8 that signal envelopes are generated roughly around $\pi / 4$ and $5 \pi / 4$ in all experiments. The variable Pha measured in the experiment $\mathrm{C}$ exhibits a lower standard deviation. In experiments $A$ and $B$ we can see that the spread of variable Pha is small, due to the short duration of the experiment (twenty minutes, capturing three periods per minute). Longer experiments would confirm a larger Pha spreading due to space charge accumulation.

Figure 8 also shows that the envelope width exhibits a different behavior depending on the semi-period of the power voltage where the PD was generated.

An interesting result is shown in Figure 9, where the envelope width $(\sigma)$ is drawn versus the rising slope $(R S)$. In this diagram we can easily discriminate the results of the four experiments. Measured results in experiments A and B (PD in the intentionally degraded area) exhibit higher rising slopes $(R S>100 \mathrm{~V} / \mu \mathrm{s})$. In the extreme case, results obtained in experiment $\mathrm{C}$ show the lowest rising slopes. Note that $R S$ values measured in experiment $B$ are greater that the values obtained in experiment $\mathrm{A}$.

Finally, Figure 10 presents a diagram where the measured falling slopes are depicted versus their corresponding rising

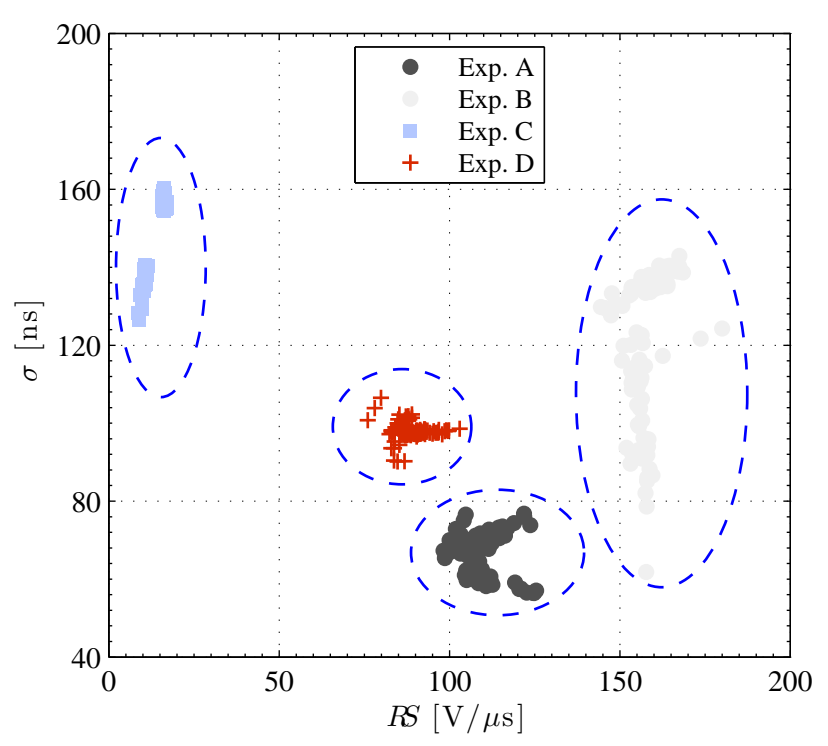

Figure 9: Envelope deviation $(\sigma) v s$. rising slope $(R S)$.

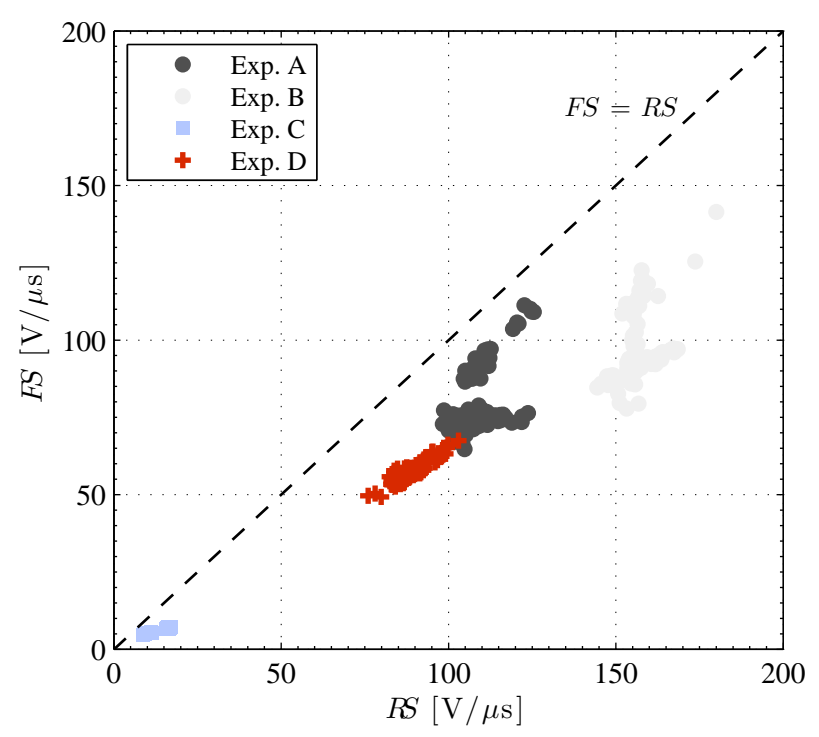

Figure 10: Envelope falling slope $(F S) v s$. rising slope $(R S)$.

slopes. These results show that we can also discriminate the type of measured phenomenon using these variables. Figure 10 confirms that the PD envelope is skewed, as the rising slope is greater than the falling slope.

\subsection{Locating the PD source}

In this case study we demonstrate that the PD source can be located by using the envelope of the signal captured at both cable ends. The proposed scheme is depicted in Figure 11 where $s_{\mathrm{CT}-1}(t)$ and $s_{\mathrm{CT}-2}(t)$ are the signals captured at current transformers CT-1 and CT-2, respectively. These signals are synchronized according to the test bench described in Figure 1.

Let $\tau_{d}$ be the difference between the times of arrival, i.e. the time that the PD envelope takes to arrive at each CT from its 
origin. The estimated PD source location $d$ fulfills the following equation: $\tau_{d}=(L-2 d) / v_{p}$, where $L$ is the cable length, and $v_{p}$, the envelope propagation velocity. In our test bench the cable length $L=340 \mathrm{~m}$ and $d \simeq 2 \mathrm{~m}$.

The envelope propagation velocity $v_{p}$ has to be known in order to estimate $d$. In this sense, some authors propose to calibrate this propagation velocity by means of measuring the propagation time of a known pulse intentionally injected in one cable end [14]. In our case, the propagation velocity has been measured using a vector network analyzer, that provides an estimation of the group velocity. Note that the group velocity is not constant versus frequency, so $v_{p}$ depends on the frequency components of the PD that propagate along the cable. Assuming the main frequency components are in the interval [1-20] $\mathrm{MHz}$ (using the CT proposed in section 2), the vector network analyzer provides a group velocity in the interval [171, 176] $\mathrm{m} / \mu \mathrm{s}$.

The dependence of $v_{p}$ on frequency is responsible for the degradation in the location estimation of techniques based on cross-correlation. This degradation is due to: 1) the estimated fault location depends on which frequency components propagate along the cable, and 2) how the cable disperses these components. It is well-known that dispersion and high frequency attenuation are responsible for degrading the location results of conventional techniques [6]. For example, dispersion is responsible for an error location of $25 \mathrm{~m}$ in a mixed power cable (PILC+XLPE) of $1540 \mathrm{~m}$ length according to reference [11].

In Figure 6 the PD signal envelope width was measured: $\sigma_{1}=68 \mathrm{~ns}$ (measured at CT-1), and $\sigma_{2}=123 \mathrm{~ns}$ (at CT-2). These results show that the original PD envelope was spread along the the cable, approximately doubling its width.

We propose the scheme of Figure 11, where the difference between the time of arrivals $\left(\tau_{d}\right)$ is estimated by calculating the maximum of the cross-correlation performed over a dispersion compensated version of the envelopes. Theoretically, the cross-correlation operation cancels the noise effects since it is uncorrelated with the signals, but in real conditions noise degrades the delay estimation performance. Especially when one of the correlated signals is spread, resulting in a flat and noisy cross-correlation function. To avoid this problem we propose in the following subsection to reshape one of the signal envelopes used to estimate $\tau_{d}$. The PD envelope captured at CT-2 is processed in the time domain in order to make it narrower before the cross-correlation operation is done.

\subsubsection{Reshaping algorithm}

In Figure $11 Q$ and $P$ factors are responsible for signal reshaping in the proposed algorithm. $Q$ is a decimation factor that narrows the resulting signal, whereas $P$ is an interpolation factor that makes the opposite. Therefore, by proper selection of $P$ and $Q$, we can control the width of the processed envelope. In order to know how much this signal should be narrowed, it is necessary to know how much the cable propagation spreads it. To this end, both signal envelope widths $\left(\sigma_{1}\right.$ and $\left.\sigma_{2}\right)$ are estimated by the standard deviation of a Gaussian function obtained by curve fitting (using the least square method). Therefore, the envelope of the signal coming from the CT-2 should

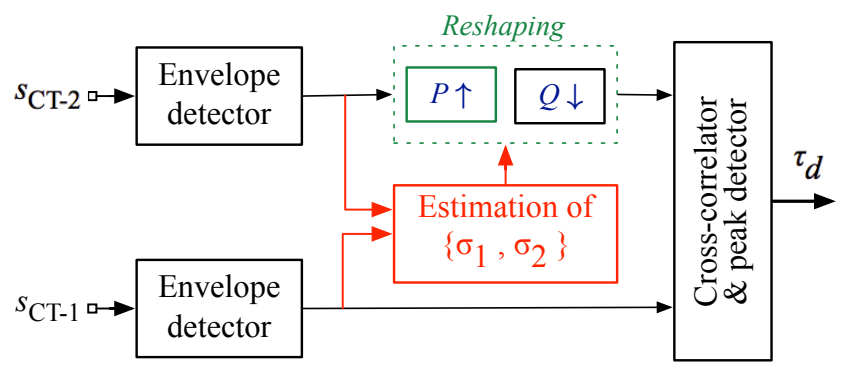

Figure 11: Diagram of the proposed location system based on estimating the time of arrival of the envelopes. One of the envelopes is previously reshaped.

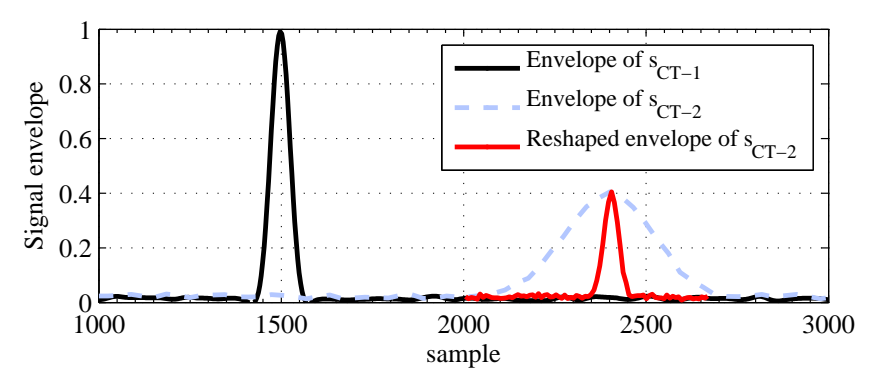

(a)

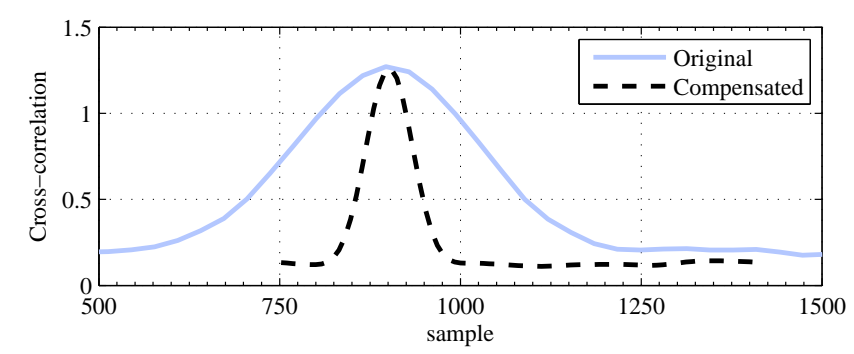

(b)

Figure 12: Operation mode of the proposed method: (a) shows the captured envelopes before and after compensation; (b) cross-correlation with and without compensation. Obtained with a $S N R=10 \mathrm{~dB}$.

be $\sigma_{2} / \sigma_{1}$ times narrower to have the same width than the signal envelope coming from CT-1. As $P$ and $Q$ must be integers, we approximate this quotient by the smallest and nearest integers: $Q / P \approx \sigma_{2} / \sigma_{1}$.

In order to explain the operation mode of the proposed technique, Figure 12 shows a simulation of the proposed technique using two Gaussian envelopes, one of them six times wider than the other one. Both envelopes simulate the signals captured in CT-1 (labeled as $s_{\mathrm{CT}-1}$ ) and CT-2 (labeled as $s_{\mathrm{CT}-2}$ ). The same power of Additive White Gaussian Noise (AWGN) is added at both input signals. The selected signal-to-noise ratio $(S N R)$ measured at CT-2 is $S N R=10 \mathrm{~dB}$ (in this simulation the $S N R$ in the CT-1 is $14 \mathrm{~dB}$ higher). The envelope of signal $s_{\mathrm{CT}-2}$ is reshaped using $Q=18$ and $P=3$. Consequently, see Figure 12(b), the cross-correlation function obtained with the reshaped version of the envelope of $s_{\mathrm{CT}-2}$ provides an more abrupt maximum. This effect is particularly important in noisy 


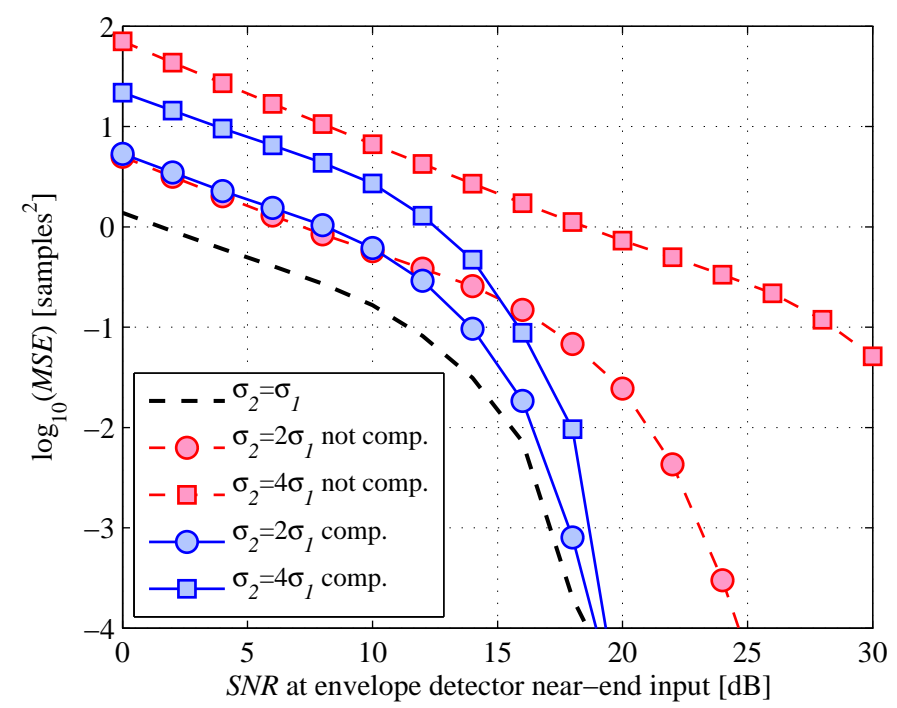

Figure 13: Mean Squared Error (MSE) of the location error (in samples) versus signal-to-noise ratio $(S N R)$.

environments as we will demonstrate next.

The features of the proposed technique have been evaluated through simulation of the effects of dispersion in Gaussian envelopes under variable noise conditions. Simulation results are shown in terms of mean squared error (MSE) versus the SNR measured at $s_{\mathrm{CT}-2}$ signal (see Figure 13). Results show a performance degradation of the location algorithm based on cross-correlation in the two cases analyzed: $\sigma_{2}=2 \sigma_{1}$, and $\sigma_{2}=4 \sigma_{1}$. Defining a quasi error free $S N R\left(S N R_{\mathrm{QEF}}\right)$ as the minimum $S N R$ required to have a $M S E<10^{-4}$, then we can see that $S N R_{\mathrm{QEF}}=20 \mathrm{~dB}$ when no dispersion is present, and $S N R_{\mathrm{QEF}}=25 \mathrm{~dB}$ if the envelope width is enlarged to twice the original size $\left(\sigma_{2}=2 \sigma_{1}\right)$. The worst case simulated, $\sigma_{2}=4 \sigma_{1}$, shows a huge degradation and exhibits a $S N R_{\mathrm{QEF}}>30 \mathrm{~dB}$. When using the proposed technique to compensate for the dispersion, the $S N R_{\mathrm{QEF}}$ is reduced to the same value as obtained with no dispersion. Focusing in low $S N R$ values, the improvement is significant only for large values of dispersion.

\subsubsection{Experimental results}

To compute the estimated fault distance $\left(d_{e}\right)$ we have measured the cable propagation velocity with a vector network analyzer. We use a mean value of $v_{p}=173 \mathrm{~m} / \mu \mathrm{s}$. This propagation speed was also obtained in $[3,4,5]$, where PD propagation characteristics were studied.

Table 3 shows the standard deviation of the error in terms of number of samples, the difference between the arrival times $\left(\tau_{d}\right)$, and finally, the distance $\left(d_{e}\right)$. In this experiment, the proposed reshaping technique improves the fault location systems based on cross-correlation. In terms of distance, we can conclude that the fault distance can be estimated with a standard deviation of less than $1 \mathrm{~m}$, instead of a standard deviation of $6.2 \mathrm{~m}$ when the dispersion is not compensated.

Experimental results show that the width envelope estimated in CT-2 roughly doubles the envelope measured in CT-1. Ac-
Table 3: Standard deviation of the estimation error: number of samples, delay, and distance.

\begin{tabular}{lccc} 
Dispersion & Samples & $\tau_{d}^{*}$ & $d_{e}^{* *}$ \\
\hline Not compensated: & 9.05 & $36.20 \mathrm{~ns}$ & $6.2 \mathrm{~m}$ \\
Compensated & 1.47 & $5.88 \mathrm{~ns}$ & $1.0 \mathrm{~m}$
\end{tabular}

* Sample time $T_{s}=4 \mathrm{~ns}$.

** Envelope propagation speed $v_{p}=173 \mathrm{~m} / \mu \mathrm{s}$.

cording to the simulation results shown in Figure 13, the location error clearly improves when the proposed technique is used to compensate a significant width difference in the envelope signal (see case $\sigma_{2}=2 \sigma_{1}$ ). But note that, in that case, the degraded area is located close to one of the cable ends. The difference shown in Table 3 between the results obtained with the compensated and not compensated signals would be smaller otherwise.

In order to generate the results of Table 3 we analyzed the PDs obtained in an experiment with a duration of one hour, capturing one power phase period each $20 \mathrm{~s}$ (3 captures per minute). After estimating the pulse widths $\sigma_{1}$ and $\sigma_{2}$ of the PDs detected, we realized that this deviation depends to a minor extent on the semi-period of the power voltage where the PDs were generated. We can presume that both the propagating conditions of PD and the initial PD characteristics are slightly different depending on the semi-period where the PD was generated. Measures of the envelope width $\sigma_{1}$ of the PDs generated in the first semi-period are greater $\left(E\left[\sigma_{1}\right]=71.08 n s\right)$ than the PDs generated in the second semi-period $\left(E\left[\sigma_{1}\right]=64.09 n s\right)$. Consequently, different pairs of factors $P$ and $Q$ have been used to tune the decimator and the interpolator in the reshaping block: $P=13$ and $Q=21$ for the first semi-period of the power voltage, and $P=11$ and $Q=18$ for the second one.

\section{Conclusions}

In this paper we propose the analysis of the partial discharge phenomenon by means of its envelope, including a new technique to reduce the negative effects of dispersion in the PD location.

The characterization of the partial discharge envelope in the time domain allows to distinguish among different types of PD that seem identical using so-called phase resolved PD patterns. The proposed variables, along with other conventional ones, permit on-line assessment of the MV cable.

This paper also illustrates that the envelope can be used to estimate the location of the partial discharge source. These results have been obtained using a new algorithm to reshape the PD envelopes before performing cross-correlation. Simulation results have shown that the proposed reshaping algorithm, based on decimating and interpolating the captured signals before correlation, improves the location algorithm performance when PD envelopes are dispersed along the cable. Experimental results performed here have shown that the fault distance can be estimated with a standard deviation of $1.0 \mathrm{~m}$. To better 
tune the proposed reshaping technique, the PD envelopes originated during the first and the second semi-period of the power voltage are separately processed with different decimation and interpolation coefficients.

\section{References}

[1] F. Mohamed, W. Siew, J. Soraghan, S. Strachan, J. McWilliam, The use of power frequency current transformers as partial discharge sensors for underground cables, IEEE Trans. Dielectr. Electr. Insul. 20 (3) (2013) 814-824. doi:10.1109/TDEI.2013.6518951.

[2] J. P. Steiner, P. Reynolds, W. Weeks, Estimating the location of partial discharges in cables, IEEE Trans. Dielectr. Electr. Insul. 27 (1) (1992) 44-59. doi:10.1109/14.123440.

[3] C.-K. Jung, J.-T. Kim, J.-B. Lee, Partial discharge simulation and analysis based on experiment in underground distribution power cables, J. Electr. Eng. Technol. 8 (4) (2013) 832-839. doi:10.5370/JEET.2013.8.4.832.

[4] Y. Md Thayoob, A. Ariffin, S. Sulaiman, Analysis of high frequency wave propagation characteristics in medium voltage xlpe cable model, in: Int. Conf. on Computer Applications and Industrial Electronic (ICCAIE), 2010, pp. 665-670. doi:10.1109/ICCAIE.2010.5735018.

[5] E. Ouatah, S. Megherfi, K. Haroun, Y. Zebboudj, Characteristics of partial discharge pulses propagation in shielded power cable, Electr. Pow. Syst. Res. 99 (0) (2013) 38 - 44. doi:10.1016/j.epsr.2013.01.012.

[6] E. Shu, S. Boggs, Effect of dispersion on pd pulse propagation in shielded power cable, in: Conf. Electrical Insulation and Dielectric Phenomena, 2008, pp. 427-430. doi:10.1109/CEIDP.2008.4772878.

[7] High-voltage test techniques. partial discharge measurement, IEC 602702000.

[8] Guide for field testing and evaluation of the insulation of shielded power cable systems, IEEE Std. 400-2002.

[9] E. Lemke, T. Strehl, W. Weissenberg, J. Herron, Practical experiences in on-site pd diagnosis tests of hv power cable accessories in service, in: IEEE Int. Symp. on Electr. Insul., 2006, pp. 498-501. doi:10.1109/ELINSL.2006.1665365.

[10] R.-N. Wu, C.-K. Chang, The use of partial discharges as an online monitoring system for underground cable joints, IEEE Trans. Power Del. 26 (3) (2011) 1585-1591. doi:10.1109/TPWRD.2011.2124474.

[11] C. Herold, T. Leibfried, Advanced signal processing and modeling for partial discharge diagnosis on mixed power cable systems, IEEE Trans. Dielectr. Electr. Insul. 20 (3) (2013) 791-800. doi:10.1109/TDEI.2013.6518949.

[12] S. Boggs, G. Stone, Fundamental limitations in the measurement of corona and partial discharge, IEEE Trans. Electr. Insul. EI-17 (2) (1982) 143-150. doi:10.1109/TEI.1982.298548.

[13] S. Boggs, J. Densley, Fundamentals of partial discharge in the context of field cable testing, IEEE Electr. Insul. Mag. 16 (5) (2000) 13-18. doi:10.1109/57.87141.

[14] R. Papazyan, R. Eriksson, Calibration for time domain propagation constant measurements on power cables, IEEE Trans. Instrum. Meas. 52 (2) (2003) 415-418. doi:10.1109/TIM.2003.811657. 